\title{
Evaluation of Muscle Chemical Composition, Amino Acids Profile and Antioxidative Capacity of the Shaziling Pig and its Crossbreeds
}

\begin{abstract}
Background: Meat quality in pigs is an extremely important economic trait. The Shaziling pig is representative of good meat quality but has been scarcely utilized because of the unpleasant growth rate and lean percentage.

Methods: The differences of muscle chemical composition, amino acids profile and antioxidative capacity were evaluated among [(Berkshire $\times$ Shaziling $) \times($ Berkshire $\times$ Shaziling $)(B S \times(B \times S)]$, (Berkshire $\times$ Shaziling $) \times$ Shaziling $[(B \times S) \times S]$, Shaziling $\times$ (Berkshire $\times$ Shaziling) $[S \times(B \times S)]$ and Shaziling $(S \times S)$ pigs

Result: Four groups had plentiful contents of mineral elements ( $\mathrm{Ca}, \mathrm{Zn}, \mathrm{Na}, \mathrm{K}, \mathrm{Cu}, \mathrm{Mg}, \mathrm{Mn}$ ) and abundant amino acids content and no obvious differences in diameters of myofibers and adipocytes were found among four groups. In addition, $(B \times S) \times S$ pigs had the highest crude protein content, possessed comprehensive nutrient in amino acids and showed preferable antioxidative capacity, suggesting that the meat of $(B \times S) \times S$ pigs has optimal shelf life.
\end{abstract}

Key words: Amino acids profile, Antioxidative capacity, Crossbreeds, Muscle chemical composition, Shaziling pigs.

\section{INTRODUCTION}

Meat quality in pigs is an extremely important economic trait for breeders, food developers and consumers. With the development of society and the promotion of people's life quality, the focal point of meat consumption demand has gradually altered from 'quantity' to 'quality' (llavarasan and Abraham 2018). The Shaziling pig, an indigenous Chinese breed mainly reared in Hunan province, is representative of good meat quality and strong adaptability (Yang et al., 2016; Chen et al., 2020). However, the Shaziling pig has been scarcely utilized on commercial farms because of the unpleasant traits of growth rate and lean percentage. Nowadays, there is a growing trend to improve the efficiency of commercial pig production by cultivating new breeds with the advantages of indigenous and lean-type pig breeds through crossbreeding systems (Jiang et al., 2011; Gopinathan et al., 2011; Guo et al., 2017).

Our previous study cultivated the crossbreeds of [Berkshire $\times$ Shaziling $) \times($ Berkshire $\times$ Shaziling $)(B S \times(B \times$ $S)$ ], (Berkshire $\times$ Shaziling $) \times$ Shaziling $[(B \times S) \times S]$, Shaziling $\times($ Berkshire $\times$ Shaziling $)[S \times(B \times S)]$ and the pure Shaziling pigs $(S \times S)$, respectively. The object of the present research was to evaluate the differences of muscle chemical composition, amino acids profile and antioxidative capacity among four groups were evaluated. This study provides the scientific basis for exploitation of pork products.

\section{MATERIALS AND METHODS}

The experiment was performed in accordance with the guidelines for the Animal Care and Use Committee of Hunan Institute of Animal and Veterinary Science in 2020.
Hunan Institute of Animal and Veterinary Science, Changsha 410131, P.R. China.

Corresponding Author: Y.L. Peng, Hunan Institute of Animal and Veterinary Science, Changsha 410131, P.R. China.

Email: 2004chch@163.com

How to cite this article: Chen, C., Liu, Y.Y., Li, H.L., Zuo, J.B., Yu, G.J. and Peng, Y.L. (2022). Evaluation of Muscle Chemical Composition, Amino Acids Profile and Antioxidative Capacity of the Shaziling Pig and its Crossbreeds. Indian Journal of Animal Research. DOI: 10.18805/IJAR.BF-1466

Submitted: 09-11-2021 Accepted: 18-12-2021 Online: 06-01-2022

\section{Animals and experimental design}

A total of 80 healthy pigs ( $50 \%$ castrated male and $50 \%$ female) with similar initial body weight were randomly selected including $B S \times(B \times S),(B \times S) \times S, S \times S$ and $S \times$ $(B \times S)$. Each group consisted of two pens with ten pigs each and the pigs were raised under similar conditions. All pigs were hand-fed two times daily and water was provided ad libitum. The feeding experiment lasted for 98 days after 7 days of adaptation period. At the end of the experiment, pigs with medium weight per pen were selected $(50 \%$ castrated male and $50 \%$ female) and the slaughter weight was recorded. Pigs were slaughtered at a local commercial slaughter house and subjected to electrical stunning, exsanguination, dehairing, then the carcass was longitudinally and symmetrically split. Longissimus thoracis (LT) and longissimus lumborum (LL) muscles on the left side of carcass were immediately harvested within 1 hour of postmortem. The ingredient and chemical composition of 
the diet offered to all pigs and the information about the experimental pigs are presented in Table 1 and 2 respectively.

\section{Muscle chemical composition}

Analysis of water in LL sample was performed in duplicate following the Association of Analytical Chemists methods (Latimer, 2012). Crude protein content was measured using Kjeldahl method (Machado et al., 2020). The method of suprapur nitric acid-hydrochloric acid digestion was utilized to detect the contents of mineral elements by ICP-OES on the basis of the Determination of Multiple Elements in Food (GB 5009.268-2016).

\section{Determination of amino acid profile}

The composition of amino acids (AAs) in LL sample was detected according to the method previously described (Liu

Table 1: Ingredient and chemical composition of experimental diet.

\begin{tabular}{lcc}
\hline Item & $\begin{array}{c}\text { Growing } \\
(30 \mathrm{~kg}-60 \mathrm{~kg})\end{array}$ & $\begin{array}{c}\text { Fattening } \\
(60 \mathrm{~kg}-)\end{array}$ \\
\hline Ingredient, \% & 50.40 & 50.50 \\
Paddy & 5.30 & 7.90 \\
Corn & 11.90 & 5.00 \\
Soybean meal & - & 10.00 \\
Rice & 6.00 & 4.60 \\
Wheat bran & 20.00 & 18.00 \\
Oil bran & 2.40 & - \\
Soybean oil & 4.00 & 4.00 \\
Premix & \\
Total & 100.00 & 100.00 \\
Chemical composition & & \\
Digestible energy, MJ kg ${ }^{-1}$ & 13.02 & 12.71 \\
Crude protein, \% & 13.51 & 11.00 \\
Calcium, \% & 0.60 & 0.62 \\
Total phosphorus, \% & 0.64 & 0.76 \\
Available phosphorus, \% & 0.18 & 0.17 \\
Lysine, \% & 0.75 & 0.60 \\
Methionine, \% & 0.22 & 0.18 \\
\hline
\end{tabular}

Supplied, per kilogram of diet: $19.8 \mathrm{mg} \mathrm{CuSO} \cdot 5 \mathrm{H}_{2} \mathrm{O} ; 0.20 \mathrm{mg} \mathrm{KI}$; $400 \mathrm{mg} \mathrm{FeSO}{ }_{4} \cdot 7 \mathrm{H}_{2} \mathrm{O} ; 0.56 \mathrm{mg} \mathrm{NaSeO} ; 359 \mathrm{mg} \mathrm{ZnSO} \cdot 7 \mathrm{H}_{2} \mathrm{O} ; 10.2$ $\mathrm{mg} \mathrm{MnSO}_{4} \cdot \mathrm{H}_{2} \mathrm{O} ; 5 \mathrm{mg}$ vitamin $\mathrm{K}$ (menadione); $2 \mathrm{mg}$ vitamin $\mathrm{B}_{1} ; 15$ mg vitamin $B_{2} ; 30 \mu g$ vitamin $B_{12} ; 5400$ IU vitamin $A ; 110$ IU vitamin $\mathrm{D}_{3} ; 18 \mathrm{IU}$ vitamin $\mathrm{E} ; 80 \mathrm{mg}$ choline chloride; $100 \mathrm{mg}$ Fungicide. et al., 2016) and the content of individual AA was represented with percentage of total amino acids.

\section{Histological analysis of muscle and adipose tissues}

The diameter of myofibers in LT samples was determined by classic hematoxylin-eosin staining. Briefly, the samples were excised perpendicularly to the direction of the myofibers and serial tissue sections of $10 \mu \mathrm{m}$ were cut using cryostat. The backfat samples with size of $10 \mathrm{~mm} \times 10 \mathrm{~mm}$ $\times 10 \mathrm{~mm}$ were snap-frozen in liquid nitrogen for subsequent Oil Red O staining. The frozen sections were washed with isopropanol and then incubated with Oil Red O solution, then counterstained with hematoxylin and mounted in glycerin jelly. All the above sections were viewed at $100 \times$ magnification using microscope and five areas were randomly selected in each sample for measuring the diameter of myofibers and adipocytes, respectively.

\section{Analysis of antioxidative parameters}

The antioxidative parameters were detected in LT and serum samples. Total superoxide dismutase (T-SOD) activity, catalase (CAT) activity and total antioxidative capacity (TAC) were analyzed by automatic biochemical analyzer as per the method of El-Baz et al., (2020). The activity of glutathione peroxidase (GSH-Px) and content of malondialdehyde (MDA) were assayed using ELISA method with automatic enzyme-mark analyzer as per the method of Qin et al., (2018). These experiments were executed by the corresponding kits following the manufacturer's protocols.

\section{Statistical analysis}

Experimental data were analyzed by one-way ANOVA procedure with SPSS 20.0 software. Duncan method was used for multiple comparison and significance test. The result was expressed as mean value and SEM and the difference was considered to be significant when $P \leq 0.05$.

\section{RESULTS AND DISCUSSION}

Muscle chemical composition

Compared with $\mathrm{BS} \times(\mathrm{B} \times \mathrm{S})$ and $\mathrm{S} \times(\mathrm{B} \times \mathrm{S})$ pigs, $(\mathrm{B} \times \mathrm{S}) \times$ $S$ and Shaziling pigs had higher $(P<0.05)$ crude protein content (Table 3 ). With regard to mineral elements, the content of Sodium in Shaziling pigs was remarkably higher $(P<0.01)$ than that in $(B \times S) \times S$ and $S \times(B \times S)$ pigs and $B S \times(B \times S)$ pigs was higher $(P<0.01)$ than $S \times(B \times S)$ pigs. And the content of Potassium in $S \times(B \times S)$ pigs were lower

Table 2: Informations about experimental pigs.

\begin{tabular}{|c|c|c|c|c|c|c|}
\hline Item & $\mathrm{BS} \times(\mathrm{B} \times \mathrm{S})$ & $(B \times S) \times S$ & $S \times S$ & $S \times(B \times S)$ & SEM & p-value \\
\hline Initial body weight, kg & 32.40 & 30.19 & 31.02 & 32.27 & 0.35 & 0.08 \\
\hline Number & 20 & 20 & 20 & 20 & & \\
\hline Slaughter weight, kg & $92.23^{a}$ & $88.30^{a}$ & $83.43^{b}$ & $90.85^{a}$ & 1.03 & $<0.01$ \\
\hline Number & 6 & 4 & 4 & 4 & & \\
\hline
\end{tabular}

BS $\times(B \times S),($ Berkshire $\times$ Shaziling $) \times($ Berkshire $\times$ Shaziling $) ;(B \times S) \times S,($ Berkshire $\times$ Shaziling $) \times$ Shaziling; $\times$ S, Shaziling $\times$
Shaziling; $S \times(B \times S)$, Shaziling $\times$ (Berkshire $\times$ Shaziling $)$.

a-e Within a row, values with different superscript letters differ $(P<0.05)$. 
$(P<0.05)$ than that in other groups (Table 2). Water is a major component of meat, accounting for approximately $75 \%$ of the meat weight (Aboagye et al., 2020), the result of this study is in line with this rule, with water content of $72.38 \%$ $73.50 \%$. Our study discovered that the content of mineral elements was plentiful and similar to the levels presented by earlier researches (Marchello et al., 1985; LombardiBoccia et al., 2005; Jukna et al., 2013; Chen et al., 2020), including Large White, Berkshire, Shaziling pigs and their crossbreeds.

\section{Amino acids profile}

Only considerable $(\mathrm{P}<0.05)$ difference in Histidine content was discovered in all detected AAs (Table 4) and Histidine content in $(B \times S) \times S$ pigs were higher $(P<0.05)$ than that in $B S \times(B \times S)$ and $S \times(B \times S)$ pigs. AAs generally take part in taste and flavors, being precursors of many odorants (Wu et al., 2018). Our research verified that aspartic acid, glutamic acid, leucine and lysine were the predominant AAs in each group, among which glutamic acid content was the

Table 3: Muscle chemical composition of $B S \times(B \times S),(B \times S) \times S, S \times S$ and $S \times(B \times S)$ pigs.

\begin{tabular}{|c|c|c|c|c|c|c|}
\hline Item & $B S \times(B \times S)$ & $(B \times S) \times S$ & $S \times S$ & $S \times(B \times S)$ & SEM & $p$-value \\
\hline Water, \% & 73.50 & 72.85 & 72.63 & 72.38 & 0.24 & 0.34 \\
\hline Crude Protein, \% & $20.47^{b}$ & $21.15^{a}$ & $21.15^{a}$ & $20.53^{b}$ & 0.11 & 0.02 \\
\hline Ash, \% & 1.05 & 1.03 & 0.99 & 1.03 & 0.01 & 0.49 \\
\hline $\mathrm{Ca}, \mathrm{mg} / \mathrm{kg}$ & 31.95 & 32.28 & 29.53 & 28.68 & 0.90 & 0.45 \\
\hline $\mathrm{Zn}, \mathrm{mg} / \mathrm{kg}$ & 16.02 & 15.65 & 15.55 & 14.70 & 0.40 & 0.72 \\
\hline $\mathrm{Na}, \mathrm{mg} / \mathrm{kg}$ & $376.00^{\mathrm{ab}}$ & $343.50^{\mathrm{bc}}$ & $387.50^{\mathrm{a}}$ & $310.00^{c}$ & 9.04 & $<0.01$ \\
\hline $\mathrm{K}, \%$ & $0.43 a$ & $0.42 a$ & $0.43 a$ & $0.40 \mathrm{~b}$ & 0.00 & 0.02 \\
\hline $\mathrm{Cu}, \mathrm{mg} / \mathrm{kg}$ & 0.43 & 0.42 & 0.49 & 0.49 & 0.02 & 0.57 \\
\hline $\mathrm{Mg}, \mathrm{mg} / \mathrm{kg}$ & 334.00 & 332.75 & 338.25 & 341.50 & 9.31 & 0.99 \\
\hline $\mathrm{Mn}, \mathrm{mg} / \mathrm{kg}$ & 0.06 & 0.06 & 0.08 & 0.08 & 0.00 & 0.11 \\
\hline
\end{tabular}

BS $\times(B \times S),($ Berkshire $\times$ Shaziling $) \times($ Berkshire $\times$ Shaziling); $(B \times S) \times S$, (Berkshire $\times$ Shaziling $) \times$ Shaziling; $S \times S$, Shaziling $\times$ Shaziling; $S \times(B \times S)$, Shaziling $\times($ Berkshire $\times$ Shaziling $)$.

a-cWithin a row, values with different superscript letters differ $(P<0.05)$.

Table 4: Amino acid profile of $B S \times(B \times S),(B \times S) \times S, S \times S$ and $S \times(B \times S)$ pigs.

\begin{tabular}{|c|c|c|c|c|c|c|}
\hline Item & $\mathrm{BS} \times(\mathrm{B} \times \mathrm{S})$ & $(B \times S) \times S$ & $S \times S$ & $S \times(B \times S)$ & SEM & $p$-value \\
\hline Aspartic acid, $\%$ & 1.91 & 1.98 & 1.90 & 1.85 & 0.02 & 0.12 \\
\hline Threonine, \% & 0.92 & 0.95 & 0.93 & 0.90 & 0.01 & 0.17 \\
\hline Serine, \% & 0.67 & 0.69 & 0.68 & 0.66 & 0.01 & 0.51 \\
\hline Glutamic acid, \% & 2.96 & 3.06 & 2.95 & 2.86 & 0.30 & 0.18 \\
\hline Glycine, \% & 0.89 & 0.91 & 0.89 & 0.85 & 0.01 & 0.17 \\
\hline Alanine, \% & 1.17 & 1.22 & 1.19 & 1.1 & 0.01 & 0.21 \\
\hline Valine, \% & 1.11 & 1.16 & 1.13 & 1.09 & 0.01 & 0.21 \\
\hline Methionine, \% & 0.53 & 0.57 & 0.53 & 0.52 & 0.01 & 0.36 \\
\hline Isoleucine, \% & 1.05 & 1.11 & 1.08 & 1.05 & 0.01 & 0.21 \\
\hline Leucine, \% & 1.74 & 1.82 & 1.77 & 1.72 & 0.02 & 0.14 \\
\hline Tyrosine, $\%$ & 0.74 & 0.78 & 0.75 & 0.73 & 0.01 & 0.12 \\
\hline Phenylalanine, \% & 0.89 & 0.95 & 0.92 & 0.90 & 0.01 & 0.09 \\
\hline Lysine, \% & 1.94 & 2.05 & 1.99 & 1.93 & 0.02 & 0.08 \\
\hline Histidine, \% & $1.00^{\mathrm{b}}$ & $1.07^{\mathrm{a}}$ & $1.02^{\mathrm{ab}}$ & $0.98^{b}$ & 0.01 & 0.03 \\
\hline Arginine, \% & 1.35 & 1.39 & 1.35 & 1.32 & 0.01 & 0.25 \\
\hline Proline, $\%$ & 0.79 & 0.83 & 0.80 & 0.76 & 0.01 & 0.09 \\
\hline Total EAAs ${ }^{\dagger}, \%$ & 9.18 & 9.66 & 9.36 & 9.08 & 0.09 & 0.09 \\
\hline Total FAAs ${ }^{\ddagger}, \%$ & 14.90 & 15.50 & 15.00 & 14.54 & 0.14 & 0.15 \\
\hline Total AAs, \% & 19.65 & 20.51 & 19.86 & 19.25 & 0.18 & 0.12 \\
\hline
\end{tabular}

BS $\times(B \times S),($ Berkshire $\times$ Shaziling $) \times($ Berkshire $\times$ Shaziling $) ;(B \times S) \times S$, (Berkshire $\times$ Shaziling $) \times$ Shaziling; $S \times S$, Shaziling $\times$ Shaziling; $S \times(B \times S)$, Shaziling $\times($ Berkshire $\times$ Shaziling $)$.

a, bWithin a row, values with different superscript letters differ $(P<0.05)$.

${ }^{\dagger}$ Total EAAs $=$ Threonine + Valine + Methionine + Isoleucine + Leucine + Phenylalanine + Lysine + Histidine.

‡Total FAAs = Aspartic acid + Serine + Glutamic acid + Glycine + Alanine + Valine + Methionine + Isoleucine + Leucine + Tyrosine + Arginine + Proline. 
highest. This finding was in accordance with former investigations obtained in several Chinese indigenous pig breeds, foreign pig breeds and their hybrid pigs and the typical hybrid pig breed Duroc $\times$ (Landrace $\times$ Large White) (Lu et al., 2008; Zhou et al., 2016; Chen et al., 2020; Chen et al., 2021), demonstrating that there is a certain rule in AAs profile in longissimus dorsi muscle of pigs. Apart from histidine, there were no significant differences in AAs among groups, including total essential amino acids (EAAs) content, total flavor amino acids (FAAs) content and total AAs content. We also found that almost all $A A s$ were higher in $(B \times S) \times S$ pigs, but lower in $S \times(B \times S)$ pigs, albeit not all the differences were to the level of statistical significance, hinting that $(B \times$ $S) \times S$ pigs possessed comprehensive nutrient in AAs.

\section{Histological analysis of muscle and adipose tissues}

As exhibited in Fig 1, there were no obvious differences in diameters of myofibers and adipocytes among four groups. It is certain that the indigenous breed Shaziling pigs are capable of depositing more fat than the lean-type Berkshire pigs and the diameter of adipocytes in backfat tissue was expectedly the highest in Shaziling pigs than that in other three groups, which is in agreement with the results acquired in other indigenous and lean-type pig breeds, such as Chinese Taihu and Landrace, Chinese Meishan and Landrace (Li et al., 2008; Nakajima et al., 2011). Meanwhile, it is noteworthy that the differences in diameter of adipocytes among four groups were not to the level of statistical significance, probably because of the Shaziling pigs consanguinity contained in three crossbreeds.

\section{Analysis of antioxidative parameters}

The activities of antioxidative enzymes in muscle and serum were estimated (Fig 2). GSH-Px, T-SOD and CAT, were the highest in $(B \times S) \times S$ pigs, but the lowest in $B S \times$ $(B \times S)$ pigs, albeit not all the differences were to the level of statistical significance. Moreover, TAC in serum was the lowest in $\mathrm{BS} \times(\mathrm{B} \times \mathrm{S})$ pigs and MDA content in both muscle and serum were, as expected, the lowest in $(B \times S) \times S$ pigs, indicating an advanced antioxidative capacity in $(B \times$ S) $\times S$ pigs. Furthermore, it has been showed that histidine indirectly participates in the antioxidative capacity of meat (Xu et al., 2017; Moro et al., 2020) and the present research revealed that histidine content in $(B \times S) \times S$ pigs was the highest. Accordingly, it was speculated that histidine content maybe another potential factor resulting in the difference in antioxidative capacity between four groups. Furthermore, our study hinted that the meat of $(B \times S) \times S$ pigs is more unsusceptible to oxidative deterioration, which is highly beneficial to the preservation of meat products (Fan et al., 2020).

B

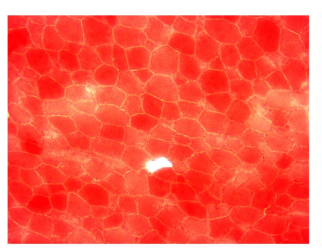

$B S \times B S$

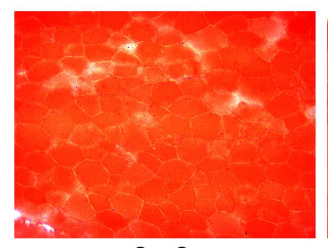

$S \times S$

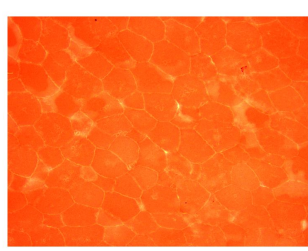

$(B \times S) \times S$

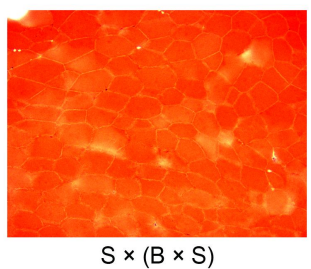

$\mathrm{S} \times(\mathrm{B} \times \mathrm{S})$

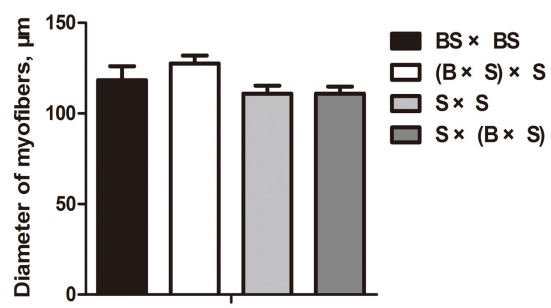

Fig 1: Histological analysis of LT muscle and backfat tissues. A. hematoxylin-eosin micrographs of myofibers (upper) and quantitative analysis of myofibers diameter (bottom) in different groups. B. Cryosectional images of adipocytes (upper) and quantitative analysis of adipocytes diameter (bottom) in different groups. 

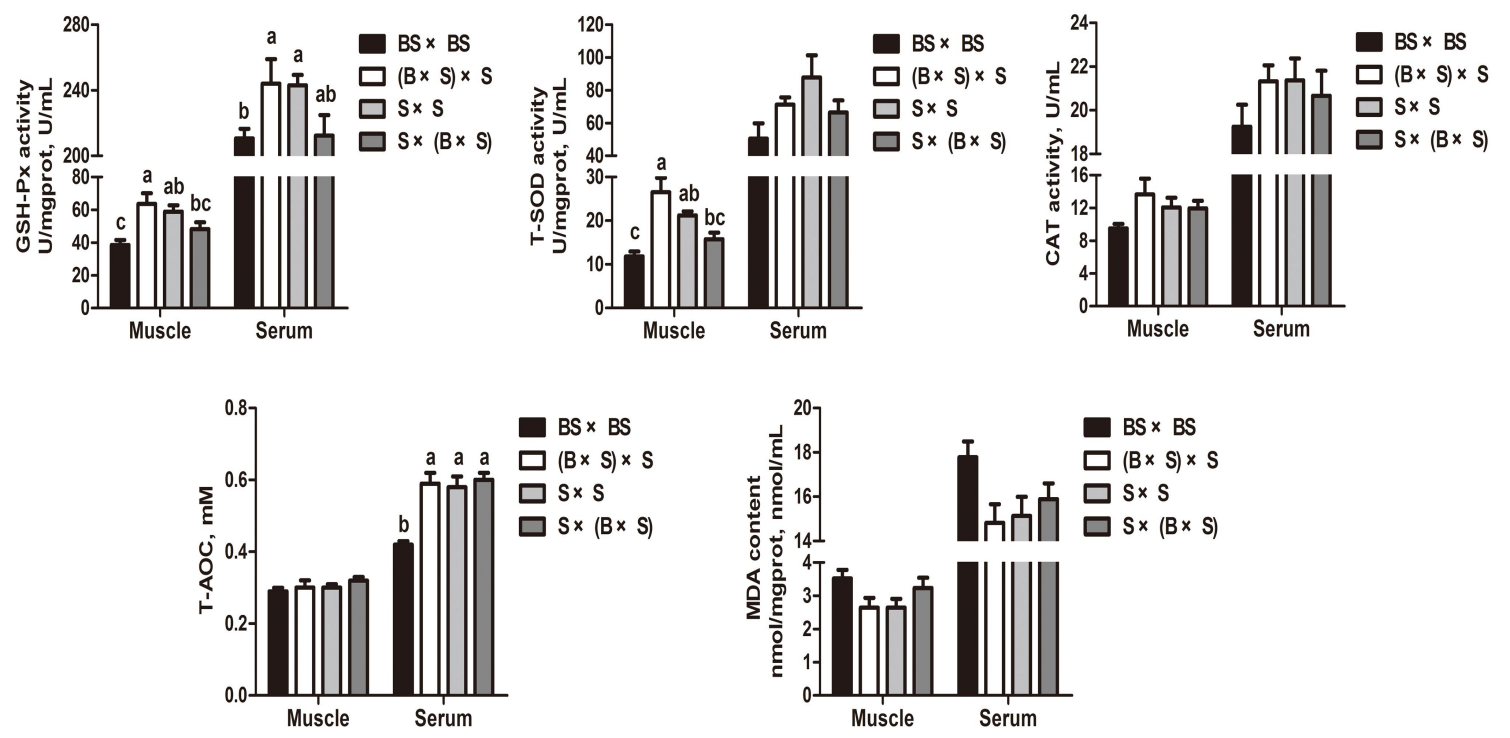

Fig 2: Analysis of antioxidative parameters in LT muscle and serum.

\section{CONCLUSION}

Four groups had plentiful content of mineral elements and abundant amino acids. $(B \times S) \times S$ pigs had highest crude protein content, possessed comprehensive nutrient in amino acids and showed preferable antioxidative capacity, suggesting that the meat of $(B \times S) \times S$ pigs has optimal shelf life.

\section{ACKNOWLEDGEMENT}

The research was supported by the Natural Science Foundation of Hunan Province (2021JJ30386), the Key Research and Development Program of Hunan Province (2020NK2024, 2019NK2193), Open Research Program of Hunan Provincial Key Laboratory (2017TP1030) and Modern Swine Industry Technology System of Hunan Province. The authors appreciate the Bureau of Agriculture and Rural Affairs of Xiangtan City, Wei Hong Food Co., Ltd. and Breeding Farm of Shaziling pigs.

\section{Conflict of interest}

The authors declare that there is no conflict of interest.

\section{REFERENCES}

Aboagye, G., Zappaterra, M., Laghi, L., Dall'Olio, S., Petracci, M., Nanni Costa, L. (2020). Water status in meat from pig breeds strongly differing in growth performances. Food Chemistry. 305: 125445.

Chen, C., Zhu, J., Ren, H.B., Deng, Y., Zhang, X., Liu, Y.Y., Cui, Q.M., Hu, X.G., Zuo, J.B., Chen, B., Zhang, X., Wu, M.S., Peng, Y.L. (2020). Growth performance, carcass characteristics, meat quality and chemical composition of the Shaziling pig and its crossbreeds. Livestock Science. 244: 104342.

Chen, G.S., Cai, Y., Su, Y.Y., Wang, D., Pan, X.L., Zhi, X.J. (2021). Study of meat quality and flavour in different cuts of Duroc-Bamei binary hybrid pigs. Veterinary Medicine and Science. 7: 724-734.
El-Baz, F.K., Salama, A., Salama R.A.A. (2020) Dunaliella salina attenuates diabetic neuropathy induced by STZ in rats: involvement of thioredoxin. BioMed Research International. 2020: 1295492.

Fan, L.J., Peng, Y., Wu, D., Hu, J.H., Shi, X.E., Yang, G.S., Li, X. (2020). Morus nigra $L$. leaves improve the meat quality in finishing pigs. Journal of Animal Physiology and Animal Nutrition (Berl). 104: 1904-1911.

Guo, Y.M., Li, L.Y., Lai, Z.S., Huang, L.B., Huang, Z.Y., Tu, J.M., Qiu, H.Q., Huang, L.S., Xiao, S.J. (2017). Present status and prospect of utilization of indigenous pig breeds in China. Acta Agriculturae Universitatis Jiangxiensis. 39: 427-435 (in Chinese).

Gopinathan, A. and Usha, A.P. (2011). Comparative evaluation of growth and carcass traits in Large White Yorkshire, Desi and their crossbred pig. Indian Journal of Animal Research. 45: 203-206.

llavarasan, R. and Abraham, J.J. (2018). The meat quality attributes and nutritional composition of three way synthetic pigs meat as influenced by age at slaughter. Indian Journal of Animal Research. 52: 464-469.

Jiang, Y.Z., Zhu, L., Li, X.W., Si, T. (2011). Evaluation of the Chinese indigenous pig breed Dahe and crossbred Dawu for growth and carcass characteristics, organ weight, meat quality and intramuscular fatty acid and amino acid composition. Animal. 5: 1485-1492.

Jukna, V., Valaitiene, V., Meskinyte-Kausiliene, E., Jankauskas, A. (2013). Comparative evaluation of large white pigs and their crossbreeds meat nutritional value and mineral content. Veterinarija ir Zootechnika. 62: 44-49.

Latimer, G.W. (2012). Official methods of analysis of AOAC International (19th ed.). Gaithersburg, MD: AOAC International.

Li, M.Z., Zhu, L., Li, X.W., Shuai, S.R., Teng, X.K., Xiao, H.S., Li, Q., Chen, L., Guo, Y.J., Wang, J.Y. (2008). Expression profiling analysis for genes related to meat quality and carcass traits during postnatal development of backfat in two pig breeds. Science in China C. Series C, Life Science. 51: 718-733. 
Liu, Y., Kong, X., Li, F., Tan, B., Li, Y., Duan, Y., Yin, Y., He, J., Hu, C., Blachier, F., Wu, G.Y. (2016). Co-dependence of genotype and dietary protein intake to affect expression on amino acid/peptide transporters in porcine skeletal muscle. Amino Acids. 48: 75-90.

Lombardi-Boccia, G., Lanzi, S., Aguzzi, A. (2005). Aspects of meat quality: Trace elements and $B$ vitamins in raw and cooked meats. Journal of Food Composition and Analysis. 18(1): 39-46.

Lu, P., Li, D.F., Yin, J.D., Zhang, L.Y., Wang, Z.Y. (2008). Flavour differences of cooked longissimus muscle from Chinese indigenous pig breeds and hybrid pig breed (Duroc $\times$ Landrace $\times$ Large White). Food Chemistry. 107: 1529-1537.

Machado, M., Machado, S., Pimentel, F.B., Freitas, V., Alves, R.C., Oliveira M.B.P.P. (2020). Amino acid profile and protein quality assessment of macroalgae produced in an integrated multi-trophic aquaculture system. Foods. 9: 1382.

Marchello, M.J., Slanger, W.D., Milene, D.B. (1985). Macro and micro minerals from selected muscles of pork. Journal of Food Science. 50: 1375-1378.

Moro, J., Tomé, D., Schmidely, P., Demersay, T.C., Azzout-Marniche, D. (2020). Histidine: A systematic review on metabolism and physiological effects in human and different animal species. Nutrients. 12: 1414.
Nakajima, I., Oe, M., Ojima, K., Muroya, S., Shibata, M., Chikuni, K. (2011). Cellularity of developing subcutaneous adipose tissue in Landrace and Meishan pigs: Adipocyte size differences between two breeds. Animal Science Journal. 82: $144-149$.

Qin, F., Pan, X.Q., Yang, J., Li, S., Shao, L., Zhang, X., Liu, B.Y., $\mathrm{Li}, \mathrm{J}$. Dietary iodine affected the GSH-Px to regulate the thyroid hormones in thyroid gland of rex rabbits. Biological Trace Element Research. 181: 251-257.

Wu, X., Xie, C.Y., Long, C.M., Li, J., Zhou, X.H., Fan, Z.Y., Blachier, F., Yin, Y.L. (2018). Effects of a daily three-meal pattern with different dietary protein contents on pig growth performance, carcass and muscle quality traits. Journal of the Science of Food and Agriculture. 98: 415-421.

Xu, N.J., Chen, G.Q., Liu, H. (2017). Antioxidative categorization of twenty amino acids based on experimental evaluation. Molecules. 22: 2066.

Yang, H., Xu, X.L., Ma, H.M., Jiang, J. (2016) Integrative analysis of transcriptomics and proteomics of skeletal muscles of the Chinese indigenous Shaziling pig compared with the Yorkshire breed. BMC Genetics. 17: 80.

Zhou, J.P., Wu, G.F., Xiang, A.Q., Wang, L., Sun, S.D., Yang, C., $\mathrm{Xu}$, F.F. (2016). Association analysis between carcass weight and meat quality of Bamei pigs. Genetics and Molecular Research. 15: 15037493. 